\title{
Single-nucleotide polymorphisms in one-carbon metabolism genes, Mediterranean diet and breast cancer risk: a case-control study in the Greek-Cypriot female population
}

\author{
Maria G. Kakkoura - Christiana A. Demetriou • Maria A. Loizidou • \\ Giorgos Loucaides · Ioanna Neophytou • Yiola Marcou • \\ Andreas Hadjisavvas $\cdot$ Kyriacos Kyriacou
}

Received: 17 April 2014/ Accepted: 5 January 2015/Published online: 21 January 2015

(C) Springer-Verlag Berlin Heidelberg 2015

\begin{abstract}
Single-nucleotide polymorphisms (SNPs) within genes of the one-carbon metabolism pathway have been shown to interact with dietary folate intake to modify breast cancer (BC) risk. Our group has previously demonstrated that the Mediterranean dietary pattern, rich in beneficial one-carbon metabolism micronutrients, protects against BC in Greek-Cypriot women. We aimed to investigate whether SNPs in the MTHFR (rs1801133 and rs1801131) and MTR (rs1805087) genes modify the effect of the Mediterranean dietary pattern on BC risk. Dietary intake data were obtained using a 32-item food-frequency questionnaire. A dietary pattern specific to the GreekCypriot population, which closely resembles the Mediterranean diet, was derived using principal component analysis (PCA) and used as our dietary variable. Genotyping
\end{abstract}

Electronic supplementary material The online version of this article (doi:10.1007/s12263-015-0453-7) contains supplementary material, which is available to authorized users.

M. G. Kakkoura - C. A. Demetriou - M. A. Loizidou ·

I. Neophytou · A. Hadjisavvas $(\bowtie) \cdot$ K. Kyriacou $(\bowtie)$

Department of EM/Molecular Pathology, The Cyprus Institute

of Neurology and Genetics, Nicosia, Cyprus

e-mail: ahsavvas@cing.ac.cy

K. Kyriacou

e-mail: kyriacos@cing.ac.cy

M. G. Kakkoura - A. Hadjisavvas - K. Kyriacou

The Cyprus School of Molecular Medicine, The Cyprus Institute

of Neurology and Genetics, Nicosia, Cyprus

G. Loucaides

The Cyprus Institute of Neurology and Genetics, Nicosia,

Cyprus

Y. Marcou

Bank of Cyprus Oncology Center, Nicosia, Cyprus was performed on subjects from the MASTOS study, a case-control study of BC in Cyprus, using TaqMan assays. Adjusted odds ratios (ORs) were estimated using logistic regression analyses. High adherence to the PCA-derived Mediterranean dietary pattern further reduced $\mathrm{BC}$ risk with increasing number of variant MTHFR 677T alleles $\left(\mathrm{OR}_{\mathrm{Q} 4 v s . \mathrm{Q} 1}\right.$ for $677 \mathrm{TT}=0.37,95 \%$ CI $0.20-0.69$, for 677 $\mathrm{CT}=0.60, \quad 95 \% \quad$ CI $0.42-0.86$ ). Additionally, high adherence to the Mediterranean dietary pattern decreased $\mathrm{BC}$ risk in subjects with at least one MTR 2756A allele $\left(\mathrm{OR}_{\mathrm{Q} 4 v s . \mathrm{Q} 1}\right.$ for $2756 \mathrm{AA}=0.59,95 \%$ CI $0.43-0.81$, for $2756 \mathrm{AG}=0.59,95 \% \mathrm{CI} 0.39-0.91)$ and in subjects with the MTHFR $1298 \mathrm{CC}$ genotype $\left(\mathrm{OR}_{\mathrm{Q} 4 \mathrm{vs} . \mathrm{Q} 1} 0.44,95 \% \mathrm{CI}\right.$ $0.30-0.65)$. Overall $P$-interaction values, however, were not statistically significant. Our study suggests that these MTHFR and MTR SNPs may act as effect modifiers, highlighting their biological significance in the association between Mediterranean diet, the one-carbon metabolism pathway and $\mathrm{BC}$.

Keywords Breast cancer - One-carbon metabolism . MTHFR · MTR · Mediterranean diet

\section{Introduction}

Breast cancer (BC) is the most prevalent form of cancer among the female population worldwide and the second most common cause of cancer related mortality in women, after lung cancer, in developed regions (GLOBOCAN 2012). It is also the most frequent form of cancer in the female population of Cyprus, with approximately 500 female $\mathrm{BC}$ cases diagnosed annually according to the National Cancer Registry. BC is a complex and multifactorial disease, which arises as a result of interactions 
between several genes and multiple environmental factors (Ponder 2001). Diet is one of the environmental factors implicated in BC risk (Vera-Ramirez et al. 2013) and epidemiological evidence suggests that a well-balanced diet, such as the Mediterranean diet, could reduce the risk of BC (Albuquerque et al. 2014; Brennan et al. 2010). The key characteristics of a Mediterranean diet are the frequent consumption of olive oil, the high intake of fruit, vegetables, legumes, cereals, bread and nuts, the moderate or low amounts of dairy products, fish, eggs and poultry, the low to moderate consumption of wine and the low amounts of red meat (Trichopoulou et al. 1995). Recently, an association study in Greek-Cypriot women showed that a dietary pattern rich in vegetables, fruit, legumes and fish, reduces the risk of post-menopausal BC (Demetriou et al. 2012). In part, this might explain the lower incidence rates of $\mathrm{BC}$ recorded in Mediterranean countries like Cyprus, compared with the rest of Europe and North America (GLOBOCAN 2012). However, the underlying molecular pathways that are involved in the interplay between Mediterranean diet and BC risk require further investigation (Vera-Ramirez et al. 2013).

One of these pathways is the one-carbon metabolism, where the biological processes of DNA methylation and DNA synthesis are interconnected (Xu and Chen 2009). Both of these processes are thought to play critical roles in carcinogenesis (Lewis et al. 2006; Maruti et al. 2009). Onecarbon metabolism requires the appropriate functioning of specific enzymes, and ample quantities of dietary micronutrients, such as folate and specific amino acids (e.g. methionine), which act as the substrates as well as other vitamins (B2, B6 and B12), which act as co-enzymes (Lucock 2000; Stevens et al. 2007). Methylenetetrahydrofolate reductase $(M T H F R)$ is a critical enzyme in the one-carbon metabolism; it catalyses irreversibly the 5,10-methylenetetrahydrofolate (5,10-methyleneTHF) to 5-methyltetrahydrofolate (5-methylTHF) (Xu et al. 2007). In turn, the 5,10-methyleneTHF facilitates the de novo synthesis of deoxy-thymidine monophosphate (dTMP) (Suzuki et al. 2008). The 5-methylTHF form can transmit one-carbon units for DNA methylation through homocysteine, methionine and S-adenosy-Lmethionine (SAM), the universal methyl donor (Choi and Mason 2002). The MTHFR gene has two functional singlenucleotide polymorphisms (SNPs), the MTHFR 677C $>\mathrm{T}$ (p.Ala222Val, rs1801133) and the MTHFR 1298A $>C$ (p.Glu429Ala, rs1801131), both of which have been shown to decrease the activity of the enzyme (Frosst et al. 1995; Weisberg et al. 2001). Another key enzyme of the one-carbon metabolism, is methionine synthase (MTR), which is responsible for catalysing the transfer of a methyl group from the 5-methylTHF to homocysteine to form a methionine (Lissowska et al. 2007). At the same time, it catalyses the de-methylation of 5-methylTHF to tetrahydrofolate (THF), which is used for the synthesis of thymidylate
(Shrubsole et al. 2006). The MTR 2756A $>$ G SNP (p.Asp919Gly, rs1805087) is the most common SNP in this gene (Ma et al. 2009a). Although the effect of this MTR SNP on the activity of the enzyme is not yet fully defined, i.e. whether it increases or decreases its activity (Lu et al. 2010; Weiner et al. 2012; Yu et al. 2010), the enzyme regulates the intracellular folate pools and maintains adequate concentrations of methionine and homocysteine, ensuring the proper functioning of the one-carbon metabolism pathway (Yu et al. 2007; Zhong et al. 2013).

Recent meta-analyses on the association between the MTHFR $677 \mathrm{C}>\mathrm{T}$ SNP and BC risk have shown conflicting results. Some of the studies have shown a positive association (Liang et al. 2013; Macis et al. 2007; Qi et al. 2010; Yu and Chen 2012; Zhang et al. 2010), whereas a number of other studies have shown no association (Lewis et al. 2006; Lissowska et al. 2007; Zintzaras 2006). Furthermore, metaanalyses on the association between MTHFR 1298A $>\mathrm{C}$ and $M T R 2756 \mathrm{~A}>\mathrm{G}$ SNPs and BC risk have shown no association (Liang et al. 2013; Lissowska et al. 2007; Lu et al. 2010; Qi et al. 2010; Qiu et al. 2011; Weiner et al. 2012; Zhong et al. 2013; Zintzaras 2006). Nevertheless, it has been suggested that interactions between MTHFR, MTR SNPs and nutrients participating in the one-carbon metabolism could influence significantly the risk of $\mathrm{BC}$, highlighting the biological importance of these associations ( $\mathrm{Xu}$ and Chen 2009). However, results are still inconclusive (Lewis et al. 2006; Ma et al. 2009a). In addition, in the majority of the previous studies, only the effect of the intake of individual nutrients and not the effect of a combination of food groups as a dietary pattern was examined (Ericson et al. 2009; Lissowska et al. 2007; Maruti et al. 2009). In this context, the Mediterranean dietary pattern, rich in plant-based foods (vegetables, fruit and legumes) and fish, is characterised by sufficient levels of folate, choline, other B vitamins and methionine, which play an important role in the one-carbon metabolism (Mas et al. 2007; Park et al. 2012; Woodside et al. 2005).

The present study assessed the association between the MTHFR $677 \mathrm{C}>\mathrm{T}$ and $1298 \mathrm{~A}>\mathrm{C}$ and the $M T R 2756 \mathrm{~A}>\mathrm{G}$ SNPs and BC risk, using subjects from the MASTOS study. In this study, we also assessed the interactions between the MTHFR and MTR SNPs and a dietary pattern that resembles the Mediterranean dietary pattern, which was reported previously (Demetriou et al. 2012) and examined the effects of their interactions on $\mathrm{BC}$ risk.

\section{Materials and methods}

Subjects

MASTOS was a population-based case-control study of $\mathrm{BC}$ in Cyprus, which recruited 1,109 female BC cases aged 
40-70 years old and 1,177 controls of the same age range. All participants were recruited between the years 2004 and 2006. Cases were women with a histologically confirmed diagnosis of BC (diagnosed between January 1999 and December 2006). Control women were females with no prior history of $\mathrm{BC}$, who participated in the national mammography population screening programme and had a negative result. Blood samples were collected from both cases and controls. More information on the purpose, design of the study, data collection and study population is described elsewhere (Hadjisavvas et al. 2010; Loizidou et al. 2010).

\section{Dietary intake assessment}

Standardised interviews were performed with each participant using a questionnaire, especially designed to collect extensive demographic and risk factor data (Demetriou et al. 2012; Hadjisavvas et al. 2010). Dietary intake was assessed using a food-frequency questionnaire (FFQ) comprising of 32 food and beverage items, through a standardised diet interview. This FFQ aimed to record the routine consumption of foods of the participants over the preceding year (for cases, this was the past 12 months prior to diagnosis). More details about the design and structure of the FFQ can be found in Demetriou et al. (2012). Principal component analysis (PCA) was previously used to investigate the dietary consumption of food items (in $\mathrm{g} /$ month) included in the FFQ and also to derive the dietary pattern that best applies to the Greek-Cypriot female population. This analysis was performed on the control subjects. Diagonal (oblimin) rotation was used to extract principal components, as orthogonal rotations failed to generate interpretable results. The adherence of subjects to each dietary pattern was estimated using a component score for each subject based on all factor loadings and the respective monthly consumption of each food. Eleven components were originally found based on an eigenvalue criterion of $>1.0$ and scree plot analysis. After interpretability of the factors of the various components, the retention of only four components was justified, as components five to eleven revealed high factor loadings on single variables. Only these four factors were thus retained to repeat the PCA. Each of the four retained components corresponded to a different dietary pattern: Pattern 1-Meat/ Potatoes, Pattern 2-Cereals/Milk/Dairy, Pattern 3-Cakes/ Sweets/Nuts/Crackers/Pasta/Rice and Pattern 4-Vegetables/Fruit/Legumes/Fish. Among the 32 food and beverage items, $23.6 \%$ of the total variance was explained by the four factors $(8.05,5.92,5.10,4.55 \%$ for the four patterns, respectively). It was concluded that the fourth dietary pattern, which included high loadings of vegetables, fruit, legumes and fish, was the one closely resembling the
Mediterranean dietary pattern. Consequently, Pattern 4 was selected as being the most appropriate to be used in the subsequent association analysis with $\mathrm{BC}$ and in the analysis with the SNPs in this study. Quartile values for adherence to this dietary pattern were determined according to the score values of the controls (Demetriou et al. 2012). Subjects in quartile one had the lowest consumption of vegetables, fruit, legumes and fish and thus lowest adherence to the PCA-derived dietary pattern and subjects in quartile four had the highest consumption of the same four food groups and therefore the highest adherence to this dietary pattern.

\section{Genotyping}

Genotyping of the MTHFR and MTR SNPs was carried out in all study participants using TaqMan SNP genotyping assays (Applied Biosystems Inc.). TaqMan Universal PCR Master Mix and 30 ng of genomic DNA were used in a final reaction volume of $5 \mu$ for each assay. Genotyping was performed using 384-well plates. The order of DNA samples from cases and controls on the 384-well plate was randomized in order to ensure that samples from cases and controls were subjected to the same study conditions. To ensure good quality control practices, $20 \%$ of the samples were genotyped in duplicate. These samples had exactly the same genotyping results. Genotyping call rates for the three SNPs ranged between 96 and $98 \%$.

\section{Statistical analysis}

A Chi-square test was carried out to examine HardyWeinberg equilibrium (HWE) in the controls. Linkage disequilibrium between the two MTHFR SNPs was calculated as D'. $P$ values, which were smaller than 0.05 $(<0.05)$, were considered to be statistically significant. Associations between each SNP and BC were investigated with the use of logistic regression. These associations were adjusted for menopausal status (pre- or post-menopausal) and age. Logistic regression was also used to assess: (a) the interactions between each SNP genotype and each quartile (1-4) of the PCA-derived Mediterranean dietary pattern on BC risk within a multiplicative model and (b) the associations between PCA-derived Mediterranean dietary pattern quartiles and BC stratified by each SNP genotype. Additionally, associations between SNP genotypes and BC risk stratified by the quartiles of the PCAderived Mediterranean dietary pattern were investigated. However, there were no additional significant associations, and thus, they are not further discussed (Supplementary Table 1). Regarding interaction analysis, multiplicative interaction terms included products of scores for SNP 
genotypes ( 0 , for homozygous wild-type genotype; 1 for homozygous variant genotype and 2, for heterozygous genotype) and dietary pattern quartiles (1, for quartile $1 ; 2$, for quartile $2 ; 3$, for quartile $3 ; 4$, for quartile 4 ). The odds ratios (ORs) and $95 \%$ confidence intervals $(\mathrm{CI})$ of $\mathrm{BC}$ risk for all associations were adjusted for menopausal status and age as well as for the other three dietary patterns of the PCA (Patterns 1, 2 and 3) that were derived previously (Demetriou et al. 2012). Age was treated as a continuous variable, and PCA-derived dietary pattern data and SNP data were treated as categorical variables in the statistical model of logistic regression. A likelihood ratio test was used to compare regression models with and without SNPs-PCA-derived Mediterranean dietary pattern interaction terms, in order to derive overall $P$-interaction values for assessing the significance of interactions between SNPs and dietary pattern in relation to BC risk. These regression models were also adjusted for menopausal status, age and for the other three dietary patterns of the PCA (Patterns 1, 2 and 3). PCA-derived Mediterranean dietary pattern was also treated as a continuous variable to assess association between this PCA-derived dietary pattern and BC, stratified by genotypes of the SNPs. These associations were adjusted further for the categorical variable of the menopausal status and for the continuous variables of age and PCA-derived dietary patterns 1, 2 and 3. Outliers were removed from this analysis. For all analyses above, additional adjustment for SNP genotypes in linkage disequilibrium with the investigated SNP did not change associations (data not shown). Statistical analysis was performed using SPSS version 21 software (SPSS, PASW Inc., Chicago, IL, USA), STATA version 11 software (StatCorp. 2007. College Station, TX, USA) and SNPStats, which is a web-based software, designed for the analysis of genetic association studies (Sole et al. 2006).

\section{Results}

Characteristics and frequencies of MTHFR and MTR SNPs

The genotype frequencies of the MTHFR $677 \mathrm{C}>\mathrm{T}$, MTHFR 1298A $>\mathrm{C}$ and MTR 2756A $>\mathrm{G}$ SNPs among the control group did not deviate from HWE (Table 1). Genotype and allele frequencies of the three SNPs in cases and controls are shown in Table 1. A considerable degree of linkage disequilibrium was observed between the MTHFR $677 \mathrm{C}>\mathrm{T}$ and MTHFR 1298A $>\mathrm{C}$ SNPs (D' $=0.9809, P$ value $<0.0001$ ), a finding consistent with other studies (Chen et al. 2005; Le Marchand et al. 2004).
Table 1 Genotype and minor allele frequencies for the MTHFR and $M T R$ single-nucleotide polymorphisms (SNPs) in the MASTOS study

\begin{tabular}{|c|c|c|}
\hline Gene/SNP & Cases & Controls \\
\hline \multicolumn{3}{|l|}{ MTHFR $677 C>T(r s 1801133)$} \\
\hline $\mathrm{C} / \mathrm{C}$ & 361 & 437 \\
\hline $\mathrm{C} / \mathrm{T}$ & 516 & 526 \\
\hline $\mathrm{T} / \mathrm{T}$ & 188 & 194 \\
\hline MAF $^{\mathrm{a}}$ & 0.42 & 0.39 \\
\hline Hardy-Weinberg $(P \text { value })^{\mathrm{b}}$ & & 0.10 \\
\hline \multicolumn{3}{|l|}{ MTHFR $1298 A>C(r s 1801131)$} \\
\hline $\mathrm{A} / \mathrm{A}$ & 138 & 150 \\
\hline $\mathrm{A} / \mathrm{C}$ & 465 & 501 \\
\hline $\mathrm{C} / \mathrm{C}$ & 468 & 486 \\
\hline MAF $^{\mathrm{a}}$ & 0.65 & 0.65 \\
\hline Hardy-Weinberg $(P \text { value })^{\mathrm{b}}$ & & 0.24 \\
\hline \multicolumn{3}{|l|}{ MTR 2756A>G (rs1805087) } \\
\hline $\mathrm{A} / \mathrm{A}$ & 679 & 684 \\
\hline $\mathrm{A} / \mathrm{G}$ & 350 & 404 \\
\hline $\mathrm{G} / \mathrm{G}$ & 45 & 68 \\
\hline MAF $^{\mathrm{a}}$ & 0.2 & 0.23 \\
\hline Hardy-Weinberg $(P \text { value })^{\mathrm{b}}$ & & 0.41 \\
\hline
\end{tabular}

MTHFR and MTR SNPs and risk of breast cancer

The associations between MTHFR and MTR SNPs and BC risk are shown in Table 2. Women homozygous for the variant MTR $2756 \mathrm{GG}$ alleles demonstrated a statistically significant decreased BC risk, when compared to the wildtype MTR 2756AA carriers, in the unadjusted model. However, when adjustments were carried out for menopausal status and age, this association lost statistical significance $(\mathrm{OR}=0.69,95 \%$ CI $0.46-1.02, P$ value $=$ 0.06). Adjusted associations between the other two SNPs studied (MTHFR 677C $>$ T and MTHFR 1298A $>$ C) and BC were not statistically significant (Table 2).

Interaction analyses between principal component analysis-derived Mediterranean dietary pattern,

MTHFR and MTR SNPs and breast cancer risk

In the interaction analyses, between each of the MTHFR and MTR SNPs and the PCA-derived Mediterranean dietary pattern, the wild-type genotype for each of the three SNPs at the lowest level of adherence to the dietary pattern (quartile 1) was used as the reference group (Table 3). None of the three SNPs studied (MTHFR $677 \mathrm{C}>\mathrm{T}$, MTHFR 1298A $>\mathrm{C}$ and MTR 2756A $>\mathrm{G}$ ) interacted significantly with the Mediterranean PCA-derived dietary 
Table 2 Odds Ratios (ORs) for the associations between breast cancer risk, MTHFR and MTR single-nucleotide polymorphisms (SNPs) in the MASTOS study

\begin{tabular}{|c|c|c|c|}
\hline $\begin{array}{l}\text { Gene/ } \\
\text { SNP }\end{array}$ & $\begin{array}{l}\text { Cases/ } \\
\text { Controls }\end{array}$ & Adjusted $^{\mathrm{b}}$ OR $(95 \% \mathrm{CI})^{\mathrm{c}}$ & $P$ value \\
\hline \multicolumn{4}{|c|}{ MTHFR $677 C>T(r s 1801133)$} \\
\hline $\mathrm{C} / \mathrm{C}$ & $361 / 436$ & 1.00 & - \\
\hline $\mathrm{C} / \mathrm{T}$ & $516 / 525$ & $1.16(0.96-1.40)$ & 0.12 \\
\hline $\mathrm{T} / \mathrm{T}$ & $188 / 193$ & $1.19(0.92-1.52)$ & $\begin{array}{l}0.18 \\
P_{\text {trend }} 0.13\end{array}$ \\
\hline \multicolumn{4}{|c|}{ MTHFR $1298 A>C(r s 1801131)$} \\
\hline $\mathrm{A} / \mathrm{A}$ & $138 / 150$ & 1.00 & - \\
\hline $\mathrm{A} / \mathrm{C}$ & $465 / 500$ & $1.03(0.79-1.35)$ & 0.83 \\
\hline $\mathrm{C} / \mathrm{C}$ & $468 / 484$ & $1.06(0.81-1.39)$ & $\begin{array}{l}0.67 \\
P_{\text {trend }} 0.97\end{array}$ \\
\hline \multicolumn{4}{|c|}{ MTR 2756A>G (rs1805087) } \\
\hline $\mathrm{A} / \mathrm{A}$ & $679 / 681$ & 1.00 & - \\
\hline $\mathrm{A} / \mathrm{G}$ & $350 / 404$ & $0.87(0.73-1.05)$ & 0.14 \\
\hline $\mathrm{G} / \mathrm{G}$ & $45 / 68$ & $0.69(0.46-1.02)$ & $\begin{array}{l}0.06 \\
P_{\text {trend }} 0.12\end{array}$ \\
\hline
\end{tabular}

${ }^{a}$ The number of cases and controls may differ from those of Table 1 due to confounder missing values

b Adjusted for menopausal status and age

c $95 \%$ CI: $95 \%$ confidence interval

pattern, since overall $P$-interaction values were not statistically significant ( $P$-interaction $0.15,0.14$ and 0.87 , respectively).

Associations between principal component analysisderived Mediterranean dietary pattern and breast cancer risk, stratified by the MTHFR and MTR SNPs genotypes

In the associations between PCA-derived nutrient pattern and $\mathrm{BC}$, when stratified by each genotype of the three SNPs, the lowest quartile (quartile 1) of adherence to PCAderived dietary pattern within each SNP genotype was used as the reference group as it is shown in Table 4. High adherence to the PCA-derived dietary pattern (quartiles 3 and 4) lowered significantly BC risk for the MTHFR 677TT women $\left(\mathrm{OR}_{\mathrm{Q} 3}\right.$ vs. $\mathrm{Q} 10.33,95 \% \mathrm{CI} 0.18-0.60$ and $\mathrm{OR}_{\mathrm{Q} 4 \text { vs. } \mathrm{Q} 1} 0.37,95 \%$ CI $0.20-0.69, P$-trend $\left.<0.0001\right)$. In addition, statistically significant decreased $\mathrm{BC}$ risk was evidenced in MTHFR 677CT women who had the highest adherence to the PCA-derived dietary pattern $\left(\mathrm{OR}_{\mathrm{Q} 4}\right.$ vs. Q1 $0.60,95 \%$ CI $0.42-0.86, P$-trend $=0.01)$. Thus, it is likely that the protective effect of the Mediterranean dietary pattern becomes stronger as the number of variant MTHFR $677 \mathrm{~T}$ alleles increases. In women who carried the variant MTHFR 1298CC genotype, high adherence to the dietary pattern (quartiles 3 and 4) reduced the risk of $\mathrm{BC}$
( $\mathrm{OR}_{\mathrm{Q} 3 \text { vs. } \mathrm{Q} 1} 0.48,95 \% \mathrm{CI} 0.33-0.70$ and $\mathrm{OR}_{\mathrm{Q} 4 \text { vs. } \mathrm{Q} 1} 0.44$, $95 \%$ CI $0.30-0.65$, P-trend <0.0001). No statistically significant associations were observed between the PCAderived pattern and $\mathrm{BC}$ risk for the carriers of wild-type alleles of both MTHFR SNPs (MTHFR 677CC or MTHFR 1298AA) (Table 4).

Additionally, high adherence to the Mediterranean dietary pattern resulted in a significantly lower risk for BC in women with the wild-type MTR 2756AA genotype $\left(\mathrm{OR}_{\mathrm{Q} 3}\right.$ vs. $\mathrm{Q} 10.68,95 \%$ CI $0.50-0.92$ and $\mathrm{OR}_{\mathrm{Q} 4}$ vs. $\mathrm{Q} 10.59$, $95 \%$ CI 0.43-0.81, P-trend <0.0001) and in women with the MTR $2756 \mathrm{AG}$ genotype $\left(\mathrm{OR}_{\mathrm{Q} 4}\right.$ vs. Q1 $0.59,95 \% \mathrm{CI}$ $0.39-0.91, P$-trend $=0.01)$. Associations between variant MTR 2756GG alleles, dietary pattern and BC were not statistically significant (Table 4).

The results for the associations between SNPs, PCAderived Mediterranean dietary pattern and $\mathrm{BC}$ risk did not differ whether the dietary pattern was treated as a categorical or as a continuous variable (Supplementary Table 2).

\section{Discussion}

This is the first study carried out in Cyprus, investigating the interactions between MTHFR and MTR SNPs and a Mediterranean dietary pattern on BC risk, as well as their effect modification on the association between this dietary pattern and $\mathrm{BC}$ risk. When examining the adjusted associations between $\mathrm{BC}$ risk and the three SNPs under study, it was shown that neither of these SNPs (MTHFR 677C $>$ T, MTHFR 1298A $>C$ and MTR $2756 \mathrm{~A}>\mathrm{G}$ ) was statistically significantly associated with $\mathrm{BC}$ risk, findings which are consistent with previous meta-analyses (Lewis et al. 2006; Liang et al. 2013; Lissowska et al. 2007; Qi et al. 2010; Qiu et al. 2011; Weiner et al. 2012; Zhong et al. 2013; Zintzaras 2006). Regarding the overall interaction analyses between each of the three SNPs studied and Mediterranean PCAderived dietary pattern, non-statistically significant overall $P$-interaction values were obtained.

However, when the associations between the Mediterranean dietary pattern and BC risk stratified by SNP genotypes were examined, high adherence to the PCA-derived dietary pattern significantly reduced BC risk in subjects with at least one MTHFR 677T allele. The effect was stronger in the homozygous variant MTHFR 677TT genotype. High adherence to the Mediterranean PCA-derived dietary pattern also lowered $\mathrm{BC}$ risk in subjects with at least one wild-type $M T R$ 2756A allele with even lower Mediterranean diet adherence (quartile 3) being protective in the homozygous wild-type MTR 2756AA genotype. Furthermore, high adherence to the Mediterranean dietary pattern by the MTHFR $1298 \mathrm{CC}$ carriers also reduced BC risk. These statistically significant 


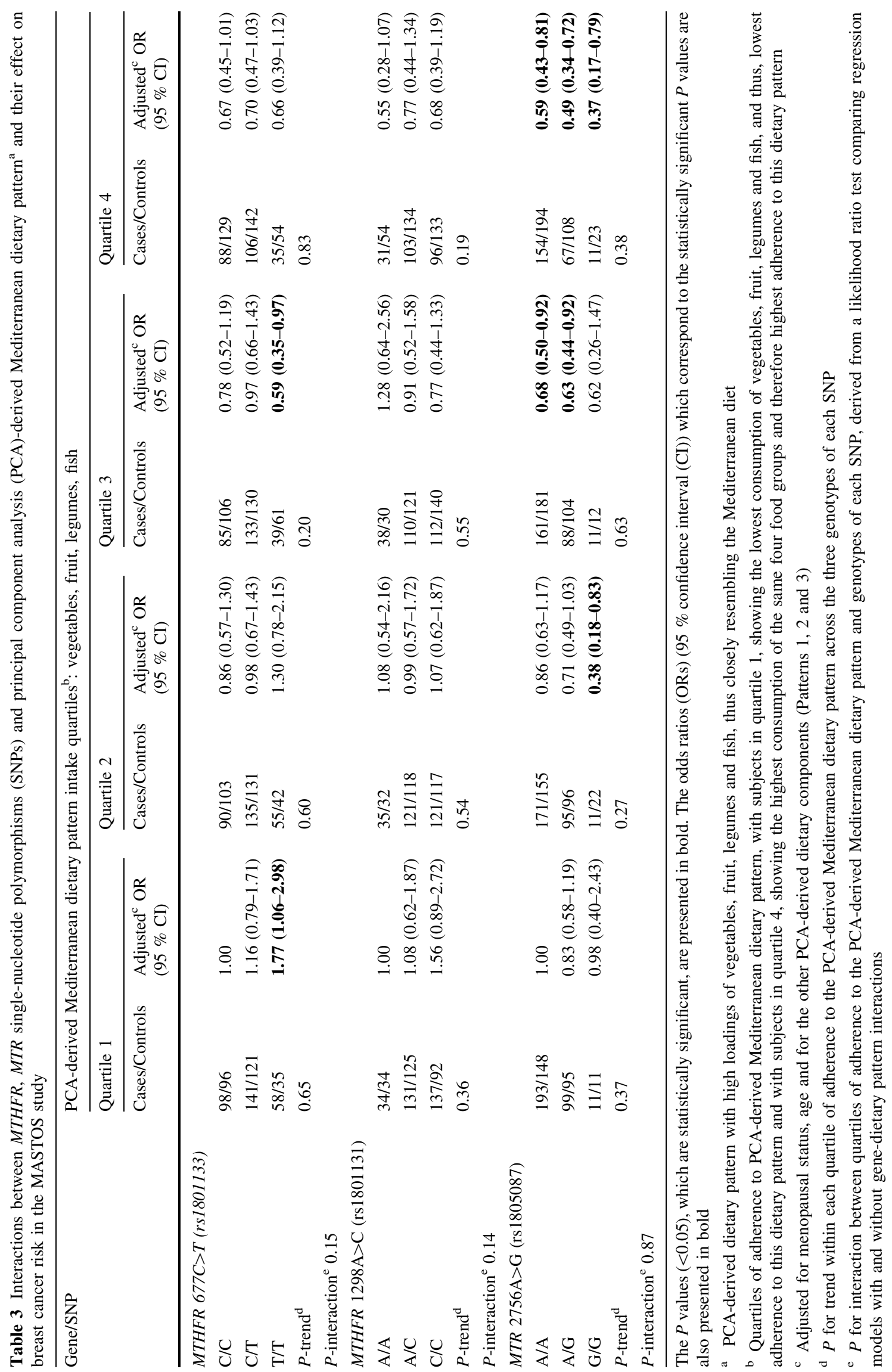


Table 4 Associations between breast cancer risk and principal component analysis (PCA)-derived Mediterranean dietary pattern ${ }^{\mathrm{a}}$, stratified by genotypes of MTHFR and MTR single-nucleotide polymorphisms (SNPs) in the MASTOS study

\begin{tabular}{|c|c|c|c|c|}
\hline Gene/SNP & $\begin{array}{l}\text { Dietary pattern } \\
\text { quartiles }{ }^{b} \text { : vegetables, } \\
\text { fruit, legumes, fish }\end{array}$ & Cases/Controls & $\begin{array}{l}\text { Adjusted }^{\mathrm{c}} \\
\text { OR }(95 \% \mathrm{CI})\end{array}$ & $P$-trend ${ }^{\mathrm{d}}$ \\
\hline \multicolumn{5}{|c|}{ MTHFR $677 C>T(r s 1801133)$} \\
\hline \multirow[t]{4}{*}{$\mathrm{C} / \mathrm{C}$} & Quartile 1 & $98 / 96$ & 1.00 & 0.04 \\
\hline & Quartile 2 & $90 / 103$ & $0.86(0.57-1.30)$ & \\
\hline & Quartile 3 & $85 / 106$ & $0.78(0.52-1.19)$ & \\
\hline & Quartile 4 & $88 / 129$ & $0.67(0.45-1.01)$ & \\
\hline \multirow[t]{4}{*}{$\mathrm{C} / \mathrm{T}$} & Quartile 1 & $141 / 121$ & 1.00 & 0.01 \\
\hline & Quartile 2 & $135 / 131$ & $0.84(0.59-1.19)$ & \\
\hline & Quartile 3 & $133 / 130$ & $0.84(0.59-1.19)$ & \\
\hline & Quartile 4 & $106 / 142$ & $0.60(0.42-0.86)$ & \\
\hline \multirow[t]{4}{*}{$\mathrm{T} / \mathrm{T}$} & Quartile 1 & $58 / 35$ & 1.00 & $<0.0001$ \\
\hline & Quartile 2 & $55 / 42$ & $0.73(0.40-1.33)$ & \\
\hline & Quartile 3 & $39 / 61$ & $0.33(0.18-0.60)$ & \\
\hline & Quartile 4 & $35 / 54$ & $0.37(0.20-0.69)$ & \\
\hline \multicolumn{5}{|c|}{ MTHFR 1298A $>C(r s 1801131)$} \\
\hline \multirow[t]{4}{*}{$\mathrm{A} / \mathrm{A}$} & Quartile 1 & $34 / 34$ & 1.00 & 0.13 \\
\hline & Quartile 2 & $35 / 32$ & $1.07(0.54-2.15)$ & \\
\hline & Quartile 3 & $38 / 30$ & $1.29(0.64-2.58)$ & \\
\hline & Quartile 4 & $31 / 54$ & $0.55(0.28-1.07)$ & \\
\hline \multirow[t]{4}{*}{$\mathrm{A} / \mathrm{C}$} & Quartile 1 & $131 / 125$ & 1.00 & 0.08 \\
\hline & Quartile 2 & $121 / 118$ & $0.91(0.63-1.31)$ & \\
\hline & Quartile 3 & $110 / 121$ & $0.86(0.59-1.24)$ & \\
\hline & Quartile 4 & $103 / 134$ & $0.73(0.50-1.05)$ & \\
\hline \multirow[t]{4}{*}{$\mathrm{C} / \mathrm{C}$} & Quartile 1 & $137 / 92$ & 1.00 & $<0.0001$ \\
\hline & Quartile 2 & $121 / 117$ & $0.68(0.47-1.00)$ & \\
\hline & Quartile 3 & $112 / 140$ & $0.48(0.33-0.70)$ & \\
\hline & Quartile 4 & $96 / 133$ & $0.44(0.30-0.65)$ & \\
\hline \multicolumn{5}{|c|}{ MTR 2756A>G (rs1805087) } \\
\hline \multirow[t]{4}{*}{$\mathrm{A} / \mathrm{A}$} & Quartile 1 & $193 / 148$ & 1.00 & $<0.0001$ \\
\hline & Quartile 2 & $171 / 155$ & $0.86(0.63-1.17)$ & \\
\hline & Quartile 3 & $161 / 181$ & $0.68(0.50-0.92)$ & \\
\hline & Quartile 4 & $154 / 194$ & $0.59(0.43-0.81)$ & \\
\hline \multirow[t]{4}{*}{$\mathrm{A} / \mathrm{G}$} & Quartile 1 & $99 / 95$ & 1.00 & 0.01 \\
\hline & Quartile 2 & $95 / 96$ & $0.86(0.57-1.29)$ & \\
\hline & Quartile 3 & $88 / 104$ & $0.76(0.51-1.15)$ & \\
\hline & Quartile 4 & $67 / 108$ & $0.59(0.39-0.91)$ & \\
\hline \multirow[t]{4}{*}{$\mathrm{G} / \mathrm{G}$} & Quartile 1 & $11 / 11$ & 1.00 & 0.25 \\
\hline & Quartile 2 & $11 / 22$ & $0.39(0.12-1.23)$ & \\
\hline & Quartile 3 & $11 / 12$ & $0.63(0.19-2.12)$ & \\
\hline & Quartile 4 & $11 / 23$ & $0.37(0.12-1.18)$ & \\
\hline
\end{tabular}

The $P$ values $(<0.05)$, which are statistically significant, are presented in bold. The odds ratios $(\mathrm{ORs})(95 \%$ confidence interval $(\mathrm{CI}))$ which correspond to the statistically significant $P$ values are also presented in bold

${ }^{a}$ PCA-derived Mediterranean dietary pattern with high loadings of vegetables, fruit, legumes and fish, thus closely resembling the Mediterranean diet

${ }^{\mathrm{b}}$ Quartiles of adherence to PCA-derived Mediterranean dietary pattern, with subjects in quartile 1, showing the lowest consumption of vegetables, fruit, legumes and fish, and thus, lowest adherence to this dietary pattern and with subjects in quartile 4, showing the highest consumption of the same four food groups and therefore highest adherence to this dietary pattern

${ }^{c}$ Adjusted for menopausal status, age and for the other PCA-derived dietary components (Pattern 1, 2 and 3)

${ }^{\mathrm{d}} P$ for trend within a specific SNP genotype across quartiles of adherence to the PCA-derived Mediterranean dietary pattern 
associations suggest that the MTHFR $677 \mathrm{C}>\mathrm{T}, M T H F R$ $1298 \mathrm{~A}>\mathrm{C}$ and $M T R 2756 \mathrm{~A}>\mathrm{G}$ SNPs could act as effect modifiers on the association between the Mediterranean dietary pattern and BC risk. Particularly, the variant MTHFR $677 \mathrm{~T}$ and the wild-type MTR 2756A alleles, as well as the homozygous variant MTHFR 1298CC genotype could enhance the protective effect of the high adherence to the Mediterranean dietary pattern, against BC risk in the GreekCypriot female population.

In contrast to other studies, which examined the influence of single micronutrients in isolation and one-carbon metabolism SNPs, our study investigated the effect of diet in terms of a dietary pattern that included a combination of various nutrients and foods. Thus, the findings of our study cannot be fully comparable with the results of previous studies. Nonetheless, an increased BC risk, in subjects with a combination of a low dietary intake of one-carbon metabolism vitamins and the MTHFR 677TT alleles, was shown in a number of earlier studies in different populations, which is in agreement with our findings (Alshatwi 2010; Chen et al. 2005; Gao et al. 2009; Lee et al. 2004; Maruti et al. 2009; Shrubsole et al. 2004). Interestingly, other studies evidenced a high BC risk in individuals with the variant MTHFR 677TT genotype and increased intake of folate (Ericson et al. 2009; Ma et al. 2009a; Stevens et al. 2007). With respect to the MTHFR 1298A >C SNP, a study found that females with the MTHFR 1298AC and MTHFR 1298CC genotype and low dietary folate consumption, had an increased BC risk, results which are similar to our results (Ma et al. 2009a). In other studies, however, no statistically significant association between dietary intake and MTHFR polymorphisms in BC was found (Le Marchand et al. 2004; Lissowska et al. 2007; Liu et al. 2013; Ma et al. 2009b; Naushad et al. 2011; Shrubsole et al. 2006; Xu et al. 2007). This discrepancy may be due to differences in several factors including ethnic background, small sample sizes, low allele frequencies or different dietary exposures (Chou et al. 2006; Ma et al. 2009a; Stevens et al. 2007).

For the association between MTR 2756A $>$ G SNP and $\mathrm{BC}$ risk, a meta-analysis which included eleven casecontrol studies showed a reduced risk of BC, in European women who had the MTR 2756GG or MTR 2756AG genotype when compared to the MTR 2756AA genotype (Lu et al. 2010). Nevertheless, stratification by menopausal status revealed no statistically significant results (Lu et al. 2010), which is in agreement with our findings. Similarly no statistically significant associations between the MTR 2756A $>$ G SNP, diet and BC risk, were revealed in the majority of the studies (Ma et al. 2009a, b; Shrubsole et al. 2006; Suzuki et al. 2008; Xu et al. 2007).

MTHFR is an important enzyme of the one-carbon metabolism, critically regulating the availability of sufficient methyl groups and the redirection of the folate pool from DNA synthesis to methylation (Maruti et al. 2009). Both the MTHFR 677TT and MTHFR 1298CC genotypes were reported to exhibit a lower enzyme activity, resulting in high total plasma homocysteine levels and low serum folate levels, mainly in the form of the 5-methylTHF when compared to homozygous wild-type genotypes (MTHFR 677CC and MTHFR 1298AA) (Ulvik et al. 2007). This is because decreased activity of the MTHFR enzyme causes an accumulation of the major form of intracellular folate, the 5,10-methyleneTHF form, that would be normally available for nucleotide synthesis, ensuring DNA stability (Chou et al. 2006; Macis et al. 2007). Although, the increased availability of 5,10-methyleneTHF in individuals with the variant MTHFR 677TT and MTHFR 1298CC SNPs would enhance DNA repair, their levels of methylated folate would be low in the event that they consume low levels of dietary folate and vitamin B2. Thus, the amount of the 5-methylTHF (major form of circulating folate in plasma) that would be available for DNA methylation would be decreased (Chou et al. 2006). In turn, this may lead to DNA hypo-methylation and activation of the expression of proto-oncogenes which may result in an increased risk of BC (Chen et al. 2005; Ma et al. 2009a; Stevens et al. 2007). On the other hand, when these individuals with the variant MTHFR (MTHFR 677TT and $M T H F R$ 1298CC) genotypes have a high adherence to a dietary pattern rich in plant-based food groups and adequate folate levels, the 5,10-methyleneTHF would be available in adequate amounts for nucleic acid synthesis (Alshatwi 2010; Choi and Mason 2002; Shrubsole et al. 2004) and there would be also enough 5-methylTHF for DNA methylation (Choi and Mason 2002). Therefore, increased dietary intake of folate and B vitamins might compensate for the deficiency in the activity of the MTHFR enzyme caused by the MTHFR $677 \mathrm{C}>\mathrm{T}$ and MTHFR 1298A >C SNPs (Chen et al. 2005; Chou et al. 2006; Xu et al. 2007). Hence, the effect of the decreased activity of the MTHFR enzyme on the biological reactions of the one-carbon metabolism could be determined by the dietary status of each individual (Alshatwi 2010).

In contrast to the biological significance assigned above to the MTHFR polymorphisms, the effect of the MTR $2756 \mathrm{~A}>\mathrm{G}$ SNP on enzyme activity is not very clear (Lu et al. 2010; Weiner et al. 2012). However, in the wild-type MTR 2756AA genotype where the enzyme retains its normal activity, high intake of methionine and folate, through a diet rich in vegetables and fish (NutritionData 2014), might increase levels of methyl groups and of intracellular folate. These high levels would favour DNA methylation events as well as nucleotide synthesis, protecting thus against cancer (Cheng et al. 2008; Lissowska et al. 2007; Yu et al. 2007). The protective effect of the 
high adherence to the PCA-derived dietary pattern in the MTR 2756AA carriers against BC risk may be explained by these anti-carcinogenic events. Nevertheless, the effect of the MTR 2756A $>$ G SNP on enzyme activity warrants further investigation.

To our knowledge, this is the first study that focuses on SNPs in genes of the one-carbon metabolism, linking BC with a dietary pattern, as opposed to a single nutrient intake. The combined intake of the food groups (vegetables, fruit, legumes and fish) included in the PCA-derived dietary pattern contains adequate levels of micronutrients, such as Vitamins B2, B6, B12, folate and choline, which are all involved in DNA methylation and synthesis (Mas et al. 2007). Indicative values for the quantities of the onecarbon metabolism nutrients in the four food groups (vegetables, fruit, legumes and fish) loaded in the PCAderived Mediterranean dietary pattern are presented in Supplementary Table 3. It is becoming increasingly recognised that in studies of healthy diets, it is more informative to evaluate the impact of a dietary pattern that represents a combination of food groups and nutrients, rather than investigating the effect of isolated micronutrients. Studying isolated nutrients could lead to misinterpretation of the results, since it does not represent the in vivo situation (Brennan et al. 2010; Caballero 2003; Gerber 2003; Velie et al. 2005; Woodside et al. 2005). Therefore, the PCA-derived dietary pattern used in this study has the advantages of closely resembling the Mediterranean diet and of being a more comprehensive approach, since it includes consumption of several micronutrients, as opposed to studies focusing on the effects of consuming single nutrients. Other strengths of the study include the large sample size, which provides sufficient statistical power to study the impact of diet on BC risk. In addition, population stratification bias is less likely in our study, since all of the participants were Greek-Cypriots, presenting a homogeneous sample in terms of ethnic background.

One probable limitation of our study is selection bias, which is a common phenomenon in case-control studies. Nevertheless, the response rate of eligible cases was $98 \%$, and the population of the study, including both cases and controls, was selected from all over the country, suggesting that selection bias in our study was minimal (Loizidou et al. 2009). Another possible limitation of the study is survival bias, since cases of the study were diagnosed with BC between 1999 and 2006 and collected between 2004 and 2006, having over a 7-year time span between the time of diagnosis, until the time of recruitment and collection of samples. Some women with the most aggressive types of BC diagnosed between 1999 and 2003 might not have been included in the study, with the possibility of introducing survival bias. However, any survival bias might be very small, since the main cancer referral centre in Cyprus, has reported a 10-year survival rate of $95 \%$ (Bank of Cyprus 2010). In addition, the FFQ used in our study examined only 32 food and beverage items, which is a limited number for a typical FFQ, without taking into account any information about dietary supplements, how each item was consumed or how it was prepared. Hence, there is a possibility that some of the missing food items might make important contributions to the dietary habits of the GreekCypriot population. Another likely limitation of the FFQ is the under-reporting in the assessment of dietary intake. Nonetheless, if a recall bias of the specific groups of foods in the PCA-derived dietary pattern is observed between cases and controls, it would be non-differential and would bias the association towards the null, since diet is not a recognised risk factor for BC (Demetriou et al. 2012). PCA has a number of limitations as well, and some of its aspects, including the variables that were derived to be included in the analysis, the number of the extracted factors, the type of the rotation used and the labelling of the retained factors, are subjective. A low amount of variance, in particular $4.55 \%$ of the total variance in the 32 items included in the FFQ, was explained by the PCA-derived component used in the present study (Demetriou et al. 2012). However, this small amount of variation is typical in dietary studies analysed by PCA, as it is shown by the limitations of reducing complex and highly connected dietary variables (Wu et al. 2009).

\section{Conclusion}

In conclusion, even though the overall $P$-interaction values were not statistically significant, our results support that the MTHFR $677 \mathrm{C}>\mathrm{T}$, MTHFR $1298 \mathrm{~A}>\mathrm{C}$ and MTR 2756A $>\mathrm{G}$ SNPs modify the effect of a high adherence to a Mediterranean dietary pattern rich in vegetables, fruit, legumes and fish, against BC risk in the Greek-Cypriot female population. In particular, an increasing number of the variant MTHFR 677T alleles conferred a slightly stronger decrease in $\mathrm{BC}$ risk of women with a high adherence to the Mediterranean dietary pattern. In addition, high adherence to the Mediterranean dietary pattern lowered BC risk in subjects with at least one wild-type MTR 2756A allele and in subjects with the MTHFR 1298CC genotype. This suggests that the high intake of the anti-carcinogenic components of the Mediterranean diet and the genetic variants of these key enzymes appear to play a synergistic role in the prevention of BC development, through the one-carbon metabolism pathway. Future work should include functional and quantitative studies targeting other enzymes [e.g. methionine synthase reductase (MTRR) and thymidylate synthase (TS)] and metabolites, which are involved in the one- 
carbon metabolism, in order to further clarify their interaction with the MTHFR and MTR enzymes as well as their role in breast carcinogenesis.

Acknowledgments This work was supported by "Cyprus Research Promotion Foundation" grants 0104/13, 0104/17 and the Cyprus Institute of Neurology and Genetics. Maria G. Kakkoura is funded by the Eurobank Cyprus Scholarship provided through the Cyprus School of Molecular Medicine. Further, we would like to thank all the study participants and acknowledge the help of the following cancer patient's organizations: the Pancyprian Association of Cancer Patients and Friends, Europa Donna Cyprus, the Cyprus Anticancer Society as well as Dr Vaios Partasides, director of the National breast cancer screening programme and his team. We also would like to express our appreciation to Doctors Eleni Kakouri, Panayiotis Papadopoulos, Maria Daniel and Simon Malas as well as to all the nurses and volunteers who provided valuable help towards the recruitment of the study participants.

Conflict of interest Maria G. Kakkoura, Christiana A. Demetriou, Maria A. Loizidou, Giorgos Loucaides, Ioanna Neophytou, Yiola Marcou, Andreas Hadjisavvas, Kyriacos Kyriacou declare that they have no conflicts of interest.

Ethical standard The study was approved by the Cyprus National Bioethics Committee. All procedures followed were in accordance with the ethical standards of the responsible committee on human experimentation (institutional and national) and with the Helsinki Declaration of 1975 , as revised in 2000. Informed consent was obtained from all patients for being included in the study.

\section{References}

Albuquerque RC, Baltar VT, Marchioni DM (2014) Breast cancer and dietary patterns: a systematic review. Nutr Rev 72:1-17. doi:10. $1111 /$ nure. 12083

Alshatwi AA (2010) Breast cancer risk, dietary intake, and methylenetetrahydrofolate reductase (MTHFR)single nucleotide polymorphisms. Food Chem Toxicol Int J Publ Br Ind Biol Res Assoc 48:1881-1885. doi:10.1016/j.fct.2010.04.028

Bank of Cyprus (2010) Bank of Cyprus Oncology Centre Registry

Brennan SF, Cantwell MM, Cardwell CR, Velentzis LS, Woodside JV (2010) Dietary patterns and breast cancer risk: a systematic review and meta-analysis. Am J Clin Nutr 91:1294-1302. doi:10.3945/ajen.2009.28796

Caballero B (2003) Fortification, supplementation, and nutrient balance. Eur J Clin Nutr 57(1):S76-S78. doi:10.1038/sj.ejen. 1601803

Chen J et al (2005) One-carbon metabolism, MTHFR polymorphisms, and risk of breast cancer. Cancer Res 65:1606-1614. doi:10. 1158/0008-5472.CAN-04-2630

Cheng CW et al (2008) Polymorphism of cytosolic serine hydroxymethyltransferase, estrogen and breast cancer risk among Chinese women in Taiwan. Breast Cancer Res Treat 111:145-155. doi:10.1007/s10549-007-9754-x

Choi SW, Mason JB (2002) Folate status: effects on pathways of colorectal carcinogenesis. J Nutr 132:2413S-2418S

Chou YC et al (2006) Genetic polymorphisms of the methylenetetrahydrofolate reductase gene, plasma folate levels and breast cancer susceptibility: a case-control study in Taiwan. Carcinogenesis 27:2295-2300. doi:10.1093/carcin/bg1108
Demetriou CA et al (2012) The mediterranean dietary pattern and breast cancer risk in Greek-Cypriot women: a case-control study. BMC Cancer 12:113. doi:10.1186/1471-2407-12-113

Ericson U, Sonestedt E, Ivarsson MI, Gullberg B, Carlson J, Olsson H, Wirfalt E (2009) Folate intake, methylenetetrahydrofolate reductase polymorphisms, and breast cancer risk in women from the Malmo diet and cancer cohort. Cancer Epidemiol Biomark Prev 18:1101-1110. doi:10.1158/1055-9965.EPI-08-0401

Frosst $\mathrm{P}$ et al (1995) A candidate genetic risk factor for vascular disease: a common mutation in methylenetetrahydrofolate reductase. Nat Genet 10:111-113. doi:10.1038/ng0595-111

Gao CM et al (2009) MTHFR polymorphisms, dietary folate intake and breast cancer risk in Chinese women. J Hum Genet 54:414-418. doi:10.1038/jhg.2009.57

Gerber M (2003) Biofactors in the Mediterranean diet. Clin Chem lab Med CCLM/FESCC 41:999-1004. doi:10.1515/CCLM.2003. 153

GLOBOCAN GCFS (2012) GLOBOCAN cancer fact sheets: breast cancer 2012. http://globocan.iarc.fr/Pages/fact_sheets_cancer. aspx

Hadjisavvas A et al (2010) An investigation of breast cancer risk factors in Cyprus: a case control study. BMC cancer 10:447. doi:10.1186/1471-2407-10-447

Le Marchand L, Haiman CA, Wilkens LR, Kolonel LN, Henderson BE (2004) MTHFR polymorphisms, diet, HRT, and breast cancer risk: the multiethnic cohort study. Cancer Epidemiol Biomark Prev 13:2071-2077

Lee SA et al (2004) Methylenetetrahydrofolate reductase polymorphism, diet, and breast cancer in Korean women. Exp Mol Med 36:116-121. doi:10.1038/emm.2004.17

Lewis SJ, Harbord RM, Harris R, Smith GD (2006) Meta-analyses of observational and genetic association studies of folate intakes or levels and breast cancer risk. J Natl Cancer Inst 98:1607-1622. doi:10.1093/jnci/djj440

Liang H, Yan Y, Li T, Li R, Li M, Li S, Qin X (2013) Methylenetetrahydrofolate reductase polymorphisms and breast cancer risk in Chinese population: a meta-analysis of 22 casecontrol studies. Tumor Biol 35:1695-1701. doi:10.1007/s13277013-1234-9

Lissowska $\mathbf{J}$ et al (2007) Genetic polymorphisms in the one-carbon metabolism pathway and breast cancer risk: a population-based case-control study and meta-analyses. Int $\mathrm{J}$ Cancer 120:2696-2703. doi:10.1002/ijc.22604

Liu Y, Zhou LS, Xu XM, Deng LQ, Xiao QK (2013) Association of dietary intake of folate, vitamin B6 and B12 and MTHFR genotype with breast cancer risk. Asian Pac J Cancer Prev APJCP 14:5189-5192

Loizidou MA et al (2009) DNA-repair genetic polymorphisms and risk of breast cancer in Cyprus. Breast Cancer Res Treat 115:623-627. doi:10.1007/s10549-008-0084-4

Loizidou MA et al (2010) Genetic variation in genes interacting with BRCA1/2 and risk of breast cancer in the Cypriot population. Breast Cancer Res Treat 121:147-156. doi:10.1007/s10549-0090518-7

Lu M, Wang F, Qiu J (2010) Methionine synthase A2756G polymorphism and breast cancer risk: a meta-analysis involving 18,953 subjects. Breast Cancer Res Treat 123:213-217. doi:10. 1007/s10549-010-0755-9

Lucock M (2000) Folic acid: nutritional biochemistry, molecular biology, and role in disease processes. Mol Genet Metab 71:121-138. doi:10.1006/mgme.2000.3027

Ma E et al (2009a) Dietary intake of folate, vitamin B6, and vitamin $\mathrm{B} 12$, genetic polymorphism of related enzymes, and risk of breast cancer: a case-control study in Brazilian women. BMC Cancer 9:122. doi:10.1186/1471-2407-9-122 
Ma E et al (2009b) Dietary intake of folate, vitamin B2, vitamin B6, vitamin B12, genetic polymorphism of related enzymes, and risk of breast cancer: a case-control study in Japan. Nutr Cancer 61:447-456. doi:10.1080/01635580802610123

Macis D et al (2007) Methylenetetrahydrofolate reductase (MTHFR) and breast cancer risk: a nested-case-control study and a pooled meta-analysis. Breast Cancer Res Treat 106:263-271. doi:10. 1007/s10549-006-9491-6

Maruti SS, Ulrich CM, Jupe ER, White E (2009) MTHFR C677T and postmenopausal breast cancer risk by intakes of one-carbon metabolism nutrients: a nested case-control study. Breast Cancer Res BCR 11:R91. doi:10.1186/bcr2462

Mas S et al (2007) Lower specific micronutrient intake in colorectal cancer patients with tumors presenting promoter hypermethylation in p16(INK4a), p4(ARF) and hMLH1. Anticancer Res 27:1151-1156

Naushad SM, Pavani A, Digumarti RR, Gottumukkala SR, Kutala VK (2011) Epistatic interactions between loci of one-carbon metabolism modulate susceptibility to breast cancer. Mol Biol Rep 38:4893-4901. doi:10.1007/s11033-010-0631-z

NutritionData (2014) Self nutrition data, know what you eat 2014: foods highest in methionine, in finfish and shellfish products. http://nutritiondata.self.com/foods-015084000000000000000.html? $\operatorname{maxCount}=200$

Park JY et al (2012) Comparison of standardised dietary folate intake across ten countries participating in the European prospective investigation into cancer and nutrition. Br J Nutr 108:552-569. doi:10.1017/S0007114511005733

Ponder BA (2001) Cancer genetics. Nature 411:336-341. doi:10. $1038 / 35077207$

Qi X et al (2010) Methylenetetrahydrofolate reductase polymorphisms and breast cancer risk: a meta-analysis from 41 studies with 16,480 cases and 22,388 controls. Breast Cancer Res Treat 123:499-506. doi:10.1007/s10549-010-0773-7

Qiu LX et al (2011) Lack of association between methylenetetrahydrofolate reductase gene A1298C polymorphism and breast cancer susceptibility. Mol Biol Rep 38:2295-2299. doi:10.1007/ s11033-010-0361-2

Shrubsole MJ et al (2004) MTHFR polymorphisms, dietary folate intake, and breast cancer risk: results from the Shanghai breast cancer study. Cancer Epidemiol Biomark Prev 13:190-196

Shrubsole MJ, Gao YT, Cai Q, Shu XO, Dai Q, Jin F, Zheng W (2006) MTR and MTRR polymorphisms, dietary intake, and breast cancer risk. Cancer Epidemiol Biomark Prev 15:586-588. doi:10.1158/1055-9965.EPI-05-0576

Sole X, Guino E, Valls J, Iniesta R, Moreno V (2006) SNPStats: a web tool for the analysis of association studies. Bioinformatics 22:1928-1929. doi:10.1093/bioinformatics/btl268

Stevens VL, McCullough ML, Pavluck AL, Talbot JT, Feigelson HS, Thun MJ, Calle EE (2007) Association of polymorphisms in one-carbon metabolism genes and postmenopausal breast cancer incidence. Cancer Epidemiol Biomark Prev 16:1140-1147. doi:10.1158/1055-9965.EPI-06-1037

Suzuki T et al (2008) One-carbon metabolism-related gene polymorphisms and risk of breast cancer. Carcinogenesis 29:356-362. doi:10.1093/carcin/bgm295

Trichopoulou A et al (1995) Diet and overall survival in elderly people. Bmj 311:1457-1460
Ulvik A, Ueland PM, Fredriksen A, Meyer K, Vollset SE, Hoff G, Schneede J (2007) Functional inference of the methylenetetrahydrofolate reductase $677 \mathrm{C}>\mathrm{T}$ and $1298 \mathrm{~A}>\mathrm{C}$ polymorphisms from a large-scale epidemiological study. Hum Genet 121:57-64. doi:10.1007/s00439-006-0290-2

Velie EM, Schairer C, Flood A, He JP, Khattree R, Schatzkin A (2005) Empirically derived dietary patterns and risk of postmenopausal breast cancer in a large prospective cohort study. Am J Clin Nutr 82:1308-1319

Vera-Ramirez L, Ramirez-Tortosa MC, Sanchez-Rovira P, RamirezTortosa CL, Granados-Principal S, Lorente JA, Quiles JL (2013) Impact of diet on breast cancer risk: a review of experimental and observational studies. Crit Rev Food Sci Nutr 53:49-75. doi:10.1080/10408398.2010.521600

Weiner AS et al (2012) Polymorphisms in the folate-metabolizing genes MTR, MTRR, and CBS and breast cancer risk. Cancer Epidemiol 36:e95-e100. doi:10.1016/j.canep.2011.11.010

Weisberg IS et al (2001) The 1298A->C polymorphism in methylenetetrahydrofolate reductase (MTHFR): in vitro expression and association with homocysteine. Atherosclerosis 156:409-415

Woodside JV, McCall D, McGartland C, Young IS (2005) Micronutrients: dietary intake v. supplement use. Proc Nutr Soc 64:543-553

Wu AH, Yu MC, Tseng CC, Stanczyk FZ, Pike MC (2009) Dietary patterns and breast cancer risk in Asian American women. Am J Clin Nutr 89:1145-1154. doi:10.3945/ajen.2008.26915

$\mathrm{Xu}$ X, Chen J (2009) One-carbon metabolism and breast cancer: an epidemiological perspective. J Genet Genomics 36:203-214. doi:10.1016/S1673-8527(08)60108-3

$\mathrm{Xu} X$ et al (2007) Polymorphisms of one-carbon-metabolizing genes and risk of breast cancer in a population-based study. Carcinogenesis 28:1504-1509. doi:10.1093/carcin/bgm061

Yu L, Chen J (2012) Association of MTHFR Ala222Val (rs1801133) polymorphism and breast cancer susceptibility: An update metaanalysis based on 51 research studies. Diagn Pathol 7:171. doi:10.1186/1746-1596-7-171

Yu CP, Wu MH, Chou YC, Yang T, You SL, Chen CJ, Sun CA (2007) Breast cancer risk associated with multigenotypic polymorphisms in folate-metabolizing genes: a nested case-control study in Taiwan. Anticancer Res 27:1727-1732

Yu K et al (2010) Methionine synthase A2756G polymorphism and cancer risk: a meta-analysis. Eur $J$ Hum Genet EJHG 18:370-378. doi:10.1038/ejhg.2009.131

Zhang J, Qiu LX, Wang ZH, Wu XH, Liu XJ, Wang BY, Hu XC (2010) MTHFR C677T polymorphism associated with breast cancer susceptibility: a meta-analysis involving 15,260 cases and 20,411 controls. Breast Cancer Res Treat 123:549-555. doi:10. 1007/s10549-010-0783-5

Zhong S, Xu J, Li W, Chen Z, Ma T, Zhao J (2013) Methionine synthase A2756G polymorphism and breast cancer risk: an upto-date meta-analysis. Gene 527:510-515. doi:10.1016/j.gene. 2013.06.054

Zintzaras E (2006) Methylenetetrahydrofolate reductase gene and susceptibility to breast cancer: a meta-analysis. Clin Genet 69:327-336. doi:10.1111/j.1399-0004.2006.00605 